\title{
Tidal disruption of white dwarfs by intermediate mass black holes
}

\author{
P. Laguna ${ }^{1}$, R. Haas ${ }^{1,2}$, R.V. Shcherbakov ${ }^{3,4}$, and T. Bode ${ }^{1}$ \\ ${ }^{1}$ Center for Relativistic Astrophysics, School of Physics, Georgia Institute of Technology, Atlanta, GA 30332, \\ USA \\ ${ }^{2}$ Theoretical AstroPhysics Including Relativity, California Institute of Technology, Pasadena, CA 91125, USA \\ ${ }^{3}$ Department of Astronomy, University of Maryland, College Park, MD 20742-2421, USA \\ ${ }^{4}$ Hubble Fellow
}

\begin{abstract}
Modeling ultra-close encounters between a white dwarf and a spinning, intermediate mass black hole requires a full general relativistic treatment of gravity. This paper summarizes results from such a study. Our results show that the disruption process and prompt accretion of the debris strongly depend on the magnitude and orientation of the black hole spin. On the other hand, the late-time accretion onto the black hole follows the same decay, $\dot{M} \propto t^{-5 / 3}$, estimated from Newtonian gravity disruption studies. The spectrum of the fallback material peaks in the soft X-rays and sustains Eddington luminosity for $1-3$ yrs after the disruption. The orientation of the black hole spin has also a profound effect on how the outflowing debris obscures the central region. The disruption produces a burst of gravitational radiation with characteristic frequencies of $\sim 3.2 \mathrm{~Hz}$ and strain amplitudes of $\sim 10^{-18}$ for galactic intermediate mass black holes.
\end{abstract}

In a tidal disruption event, the spin of black hole $(\mathrm{BH})$ will most likely not be aligned with the orbital angular momentum of the incoming star. As a consequence, the tidal debris when it falls back will form a tilted disk [1]. For thin disks, the Bardeen-Petterson effect will help align the inner part of the disk with the $\mathrm{BH}$ spin [2], but for the thick disks expected from tidal disruptions, the alignment may not happen [3-5], potentially changing our conventional picture of these explosive events. Even more remarkable will be ultra-close encounters, where an incoming star and associated tidal debris will experience general relativistic effects. Examples of strong curvature effects altering the dynamics of the disruption are frame dragging and the location of the innermost stable orbit, responsible for changing the traditional S-shape debris observed in Newtonian gravity simulations to a shell-like appearance, engulfing the $\mathrm{BH}$.

There are very few tidal disruption numerical simulations that account for general relativistic effects [69]. This paper summarizes the results from a study published recently [10]. The study was primarily aimed at investigating observational signatures associated with strong gravity that may shed light on the presence of intermediate mass BHs. It included estimates of electromagnetic transient radiation using a slim disk model to compute the spectrum during the fallback phase. We found that the sources shine at Eddington luminosity $\sim 10^{41} \mathrm{erg} \mathrm{s}^{-1}$ for about $1-3 \mathrm{yrs}$, and then fade approximately as $\propto t^{-5 / 3}$. Furthermore, there is a 50/50 chance that the inner disk will be obscured by outflowing debris for a fully misaligned $\mathrm{BH}$ spin. The study also included estimates of the gravitational wave burst signal produced during the encounters.

The study involved six simulations listed in Table 1. All had a BH with mass $M_{\mathrm{bh}}=10^{3} M_{\odot}$, and a white dwarf (WD) in a parabolic orbit with mass $M_{\mathrm{wd}}=1 M_{\odot}$ and radius $R_{\mathrm{wd}} \simeq 6,000 \mathrm{~km}$ [11]. The simulations are labeled as "BxSy" in which "Bx" gives the penetration
Table 1. Actual penetration factor $\beta^{*}$ measured from the simulations. Maximum temperature $T_{\max }$ and central density $\rho / \rho_{\text {initial }}$ with $\rho_{\text {initial }}=1.33 \times 10^{7} \mathrm{~g} \mathrm{~cm}^{-3}$. Fraction $f_{\text {acc }}$ of the star accreted during the first $2 \mathrm{~s}$ after disruption, unbound fraction $f_{\text {unb }}$ at the end of the simulation at $\sim 6 \mathrm{~s}$.

\begin{tabular}{lccccc}
\hline Run & $\beta^{*}$ & $T_{\max }\left(10^{9} \mathrm{~K}\right)$ & $\rho_{\max } / \rho_{\text {initial }}$ & $f_{\text {acc }}$ & $f_{\text {unb }}$ \\
\hline B6S0 & 8.9 & 8.6 & 7.5 & $68 \%$ & $19 \%$ \\
B6Su & 9.44 & 6.6 & 9.3 & $<1 \%$ & $60 \%$ \\
B6Sd & 11 & 12 & 3.2 & $>99 \%$ & $<0.5 \%$ \\
B6Si & 7.6 & 8.6 & 3.3 & $65 \%$ & $22 \%$ \\
B6Sa & 9.1 & 6.0 & 8.1 & $2 \%$ & $67 \%$ \\
B8Sa & 10 & 9.1 & 4.0 & $43 \%$ & $34 \%$ \\
\hline
\end{tabular}

factor $\beta=x$ used to set the initial configuration and "Sy" the $\mathrm{BH}$ spin orientation, $\mathrm{y}=(\mathrm{u}, \mathrm{d}, \mathrm{i}, \mathrm{a})$ for (up, down, inplane, arbitrary), respectively. All simulations had a $\mathrm{BH}$ with spin parameter $a / M_{\mathrm{bh}}=0.6$, except the first one which was non-spinning and the last one with $a / M_{\mathrm{bh}}=$ 0.8 . The penetration factor is defined as $\beta=R_{\mathrm{t}} / R_{\mathrm{p}}$ with $R_{\mathrm{p}}$ the pericentric distance and $R_{\mathrm{t}}$ an estimate of the tidal radius, $R_{\mathrm{t}}=R_{\mathrm{wd}}\left(M_{\mathrm{bh}} / M_{\mathrm{wd}}\right)^{1 / 3} \approx 40 M_{\mathrm{bh}}$. Also listed in Table 1 are the maximum temperatures $T_{\max }$ and densities $\rho_{\max } / \rho_{\text {initial }}$ reached by the WD before total disruption. In the second column is the "actual" value of the penetration factor. The effect of the spin orientation can be noticed by comparing the B6Su and B6Sd cases, $T_{\max }$ almost doubles and $\rho_{\max }$ triples when going from a spin aligned with the orbital angular momentum to one that is anti-aligned. This is because in the anti-aligned case B6Sd, the WD penetrates closer, than in the aligned case B6Sd, as seen from their $\beta^{*}$ values. Unfortunately, because our values of $\beta^{*}$ span a very modest range, we were not able to verify the scaling $T_{\max } \propto \beta^{2}$ and $\rho_{\max } \propto \beta^{3}$ suggested by Carter and Luminet [12]. Finally, the last two columns in Table 1 give the fraction $f_{\text {acc }}$ of the star accreted during the first $2 \mathrm{~s}$ after disruption as well as the unbound fraction $f_{\text {unb }}$ after $\sim 6 \mathrm{~s}$. 


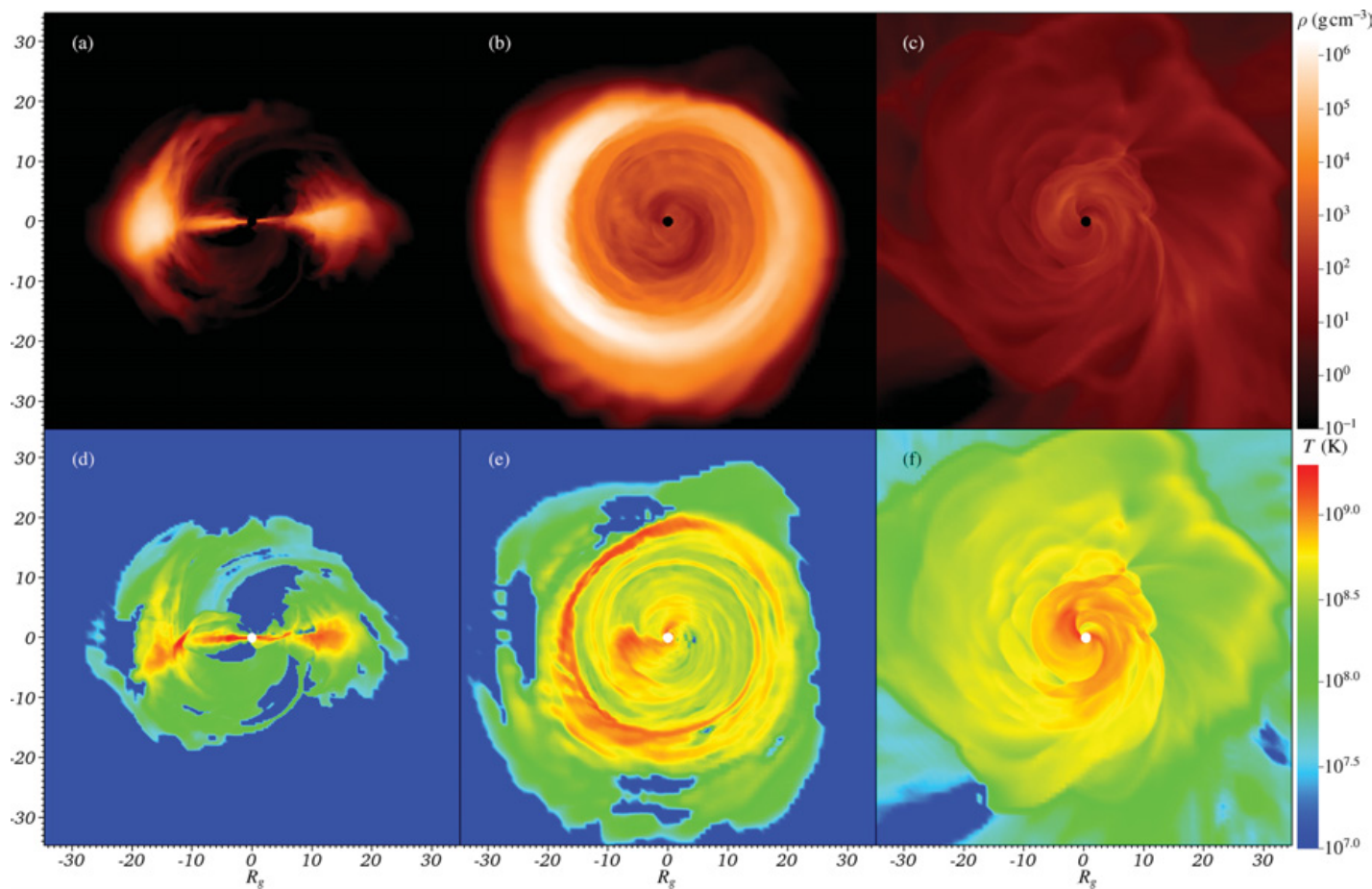

Figure 1. Snapshots in the orbital plane of the density (top row, in $\mathrm{g} \mathrm{cm}^{-3}$ ) and temperature (bottom row, in $\mathrm{K}$ ). The snapshots in the left column are for the case B6Si (spin direction in the orbital plane) $\sim 0.5$ seconds after disruption. The middle and right columns correspond to case B6Su (spin perpendicular to the orbital plane) $\sim 0.5$ seconds and $\sim 5.9$ seconds after disruption, respectively. Figure reproduced from [10].

As mentioned before, relativistic precession and frame dragging will modify the geometry of the tidal debris when compared to that from Newtonian gravity. When general relativity is included, the leading edge of the debris will wrap around the $\mathrm{BH}$ and self interact with the trailing material, creating a hot region. The spin of the $\mathrm{BH}$ will determine the location of this hot spot. If the spin is aligned with the orbital angular momentum, the hot region will be mostly contained within the equatorial plane. Figure 1 shows snapshots in the orbital plane for two of the cases investigated. The top row gives the density profiles and the bottom one the temperature. The snapshots in the left column are for the case $\mathrm{B} 6 \mathrm{Si} \sim 0.5$ seconds after disruption. The middle and right columns correspond to case $\mathrm{B} 6 \mathrm{Su} \sim 0.5$ seconds and $\sim 5.9$ seconds after disruption, respectively. $\mathrm{B} 6 \mathrm{Su}$ is a case in which the spin is aligned with the orbital angular momentum. Notice that the hot region forms a spiral which moves outwards from the point where the leading edge of the WD intersected with the tail material. At late times this feature washes out.

For the cases in which the spin of the BH is not aligned with the orbital angular momentum of the binary (i.e. B6Si, $\mathrm{B} 6 \mathrm{Sa}$ and B8Sa cases), frame dragging is not as effective in wrapping the debris material completely around the $\mathrm{BH}$ as in the B6S0, B6Su and B6Sd cases. This is because frame dragging by the $\mathrm{BH}$ in misaligned situations also pulls the material out of the orbital plane. On the other hand, the same dragging is able to spread the debris into a shell surrounding the $\mathrm{BH}$, as observed in in the left column of Figure 1 for the B6Si case in which the $\mathrm{BH}$ spin direction is perpendicular to the orbital angular momentum. These changes in the geometry of the debris are not captured by Newtonian calculations.

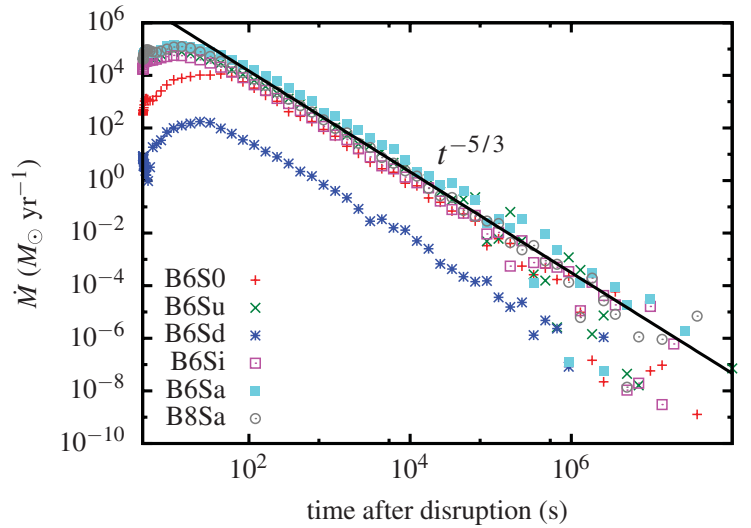

Figure 2. Mass accretion rates computed from the estimated fallback times of the fluid elements. The solid line represents a $t^{-5 / 3}$ falloff. Figure reproduced from [10].

At late times the bound tidal debris rains back onto the $\mathrm{BH}$. Our estimates yield the characteristic rate of accretion $\dot{M} \propto t^{-5 / 3}[1,13]$ as observed in Figure 2. The rates are computed from $\dot{M} \approx(d M / d \varepsilon)(d \varepsilon / d t)$, with $\varepsilon$ being the specific kinetic energy [14-16]. The term $d M / d \varepsilon$ is obtained from a snapshot at the end of the simulation, and $d \varepsilon / d t$ from geodesic integration of each fluid element assuming that the tidal debris is basically collisionless. As long as $d M / d \varepsilon \approx$ constant, the accretion rate is well approximated by $t^{-5 / 3}$. As observed in Figure 2, this takes place for times $t \gtrsim 200 \mathrm{~s}$ after the disruption.

Emission estimates in our study [10] were obtained using a slim disk model [17-20], assuming that the angular momentum of the disk is aligned with the orbital angular 


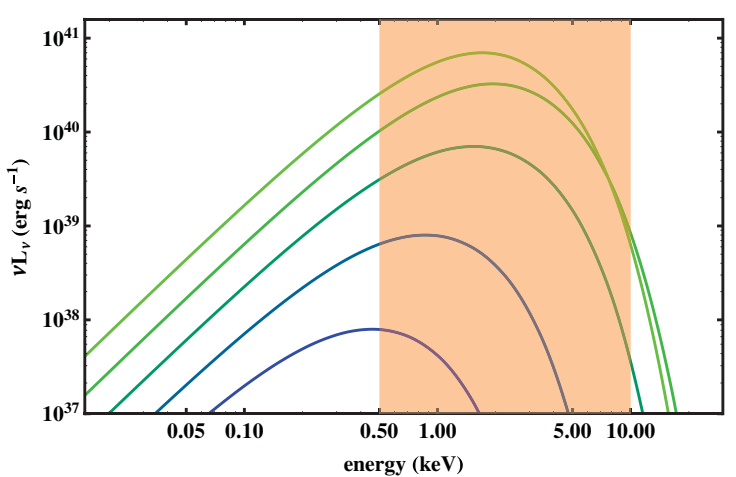

Figure 3. Spectrum for the slim disk formed following the tidal disruption at various times. The spectra shown corresponds to the case B6Su. From top to bottom: at $t=2$ months, $t=8$ months, $t=3$ years, $t=10$ years, and $t=30$ years, respectively. Figure reproduced from [10].

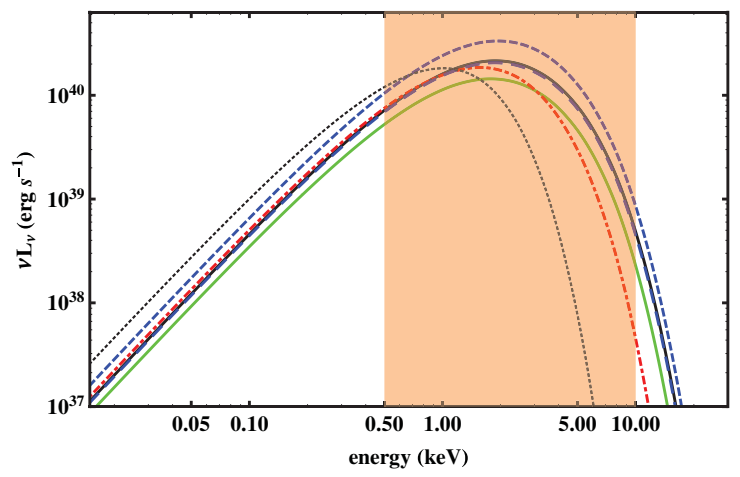

Figure 4. Spectra of our slim disk models at $t=1 \mathrm{yr}$ : B6Su case (top solid/black), B6Si case (bottom solid/green), B6Sa case (short-dashed/blue), B8Sa (long-dashed/blue), B6S0 case (dotdashed/red). Differences are due to changing spin and fallback time. Dotted line corresponds to an edge-on view of a slim disk for b6ap6 simulation. The edge-on disk has a softer spectrum and a comparable luminosity. Figure reproduced from [10].

momentum of the incoming WD. The disk will then, in principle, be misaligned with the spin of the BH. The disk spectra are shown in Figure 3. From top to bottom are $v L_{v}$ as a function of energy at 2 and 8 months, then 3, 10 and 30 years, respectively, for the case B6Su. Notice that the hard tail of the spectrum saturates at the highest accretion rates. On the other hand, the low-energy tail does not saturate at $\dot{M} \sim 4000 \dot{M}_{\text {Edd }}$. This is because the radiation time is still shorter than or comparable to the advection timescale at large distances from the $\mathrm{BH}$, where the low-energy tail is emitted. As the accretion rate drops with time, the luminosity (the area under the curve) also drops.

Figure 4 shows the disk spectra at $t=1 \mathrm{yr}$ after the disruption for all the cases studied. The spectra differ among the different cases because of two factors. The first factor is the magnitude of the accretion rate $\dot{M}$. More debris falls back onto the $\mathrm{BH}$ in the B6Sa case than in the other cases (see Figure 2). Thus, this case is systematically brighter. Similarly, the lower accretion rate for the $\mathrm{B} 6 \mathrm{Si}$ case makes its spectrum systematically weaker than the others. We found temporal degeneracies among all $a / M_{\mathrm{bh}}=0.6$ cases when the accretion rates $\dot{M}$ are the same. For example, the spectra for the B6Sa case at about $t=16$ months, for the B6Su case at $t=12$ months and for the B6Si case at about $t=9$ months are the same because at those times their accretion rates $\dot{M}$ are similar. The second reason why the spectra in Figure 4 vary is due to the changes in the radius of the innermost stable orbit.

The main conclusions from our study [10] are: 1) Independently of the orientation of the spin of the $\mathrm{BH}$, the bound tidal debris after disruption forms a thick accretion disk. 2) Obscuration of the inner region depends on the orientation of the $\mathrm{BH}$ spin and related frame-dragging. 3) The accretion flow luminosity stays around $L_{\text {Edd }}$ before $\sim 1 \mathrm{yr}$ and starts dropping as $t^{-5 / 3}$ afterwards. 4) The spectrum peaks at soft X-rays and are similar to thin disk spectra at low accretion rates $\dot{M} \ll \dot{M}_{\text {Edd }}$. 5) Selfobscuration leads to softer spectrum while obscuration by debris makes a fallback disk invisible during most of the active accretion period. 6) The gravitational wave signal depends weakly on the orientation of the spin and will be challenging to be detected for extragalactic sources.

The authors are thankful to Ramesh Narayan for fruitful discussions. RVS is supported by NASA Hubble Fellowship grant HST-HF-51298.01. RH gratefully acknowledges support by the Natural Sciences and Engineering Council of Canada. Work supported by NSF grants 0653443, 0855892, 0914553, 0941417, 0903973, 0955825. Computations at Teragrid TG-MCA08X009 and the Georgia Tech FoRCE cluster.

\section{References}

[1] M.J. Rees, Nature 333, 523 (1988)

[2] J.M. Bardeen, J.A. Petterson, Astrophys. J. 195, L65 (1975)

[3] J.C.B. Papaloizou, J.E. Pringle, MNRAS 202, 1181 (1983)

[4] P.C. Fragile, O.M. Blaes, P. Anninos, J.D. Salmonson, Ap. J. 668, 417 (2007)

[5] N. Stone, A. Loeb, Physical Review Letters 108, 061302 (2012)

[6] P. Laguna, W.A. Miller, W.H. Zurek, Ap. J. 404, 678 (1993)

[7] P. Laguna, W.A. Miller, W.H. Zurek, M.B. Davies, Ap. J. Lett. 410, L83 (1993)

[8] S. Kobayashi, P. Laguna, E.S. Phinney, P. Meszaros, Astrophys. J. 615, 855 (2004)

[9] T. Bogdanović, M. Eracleous, S. Mahadevan, S. Sigurdsson, P. Laguna, Ap. J. 610, 707 (2004)

[10] R. Haas, R.V. Shcherbakov, T. Bode, P. Laguna, Ap. J. 749, 117 (2012)

[11] T. Hamada, E.E. Salpeter, apj 134, 683 (1961)

[12] B. Carter, J.P. Luminet, Nature 296, 211 (1982)

[13] E.S. Phinney, Manifestations of a Massive Black Hole in the Galactic Center, in The Center of the Galaxy, edited by M. Morris (1989), Vol. 136 of IAU Symposium, pp. 543-+

[14] C.R. Evans, C.S. Kochanek, Ap. J. 346, L13 (1989) 
[15] G. Lodato, A.R. King, J.E. Pringle, Monthly Notices of the Royal Astronomical Society 392, 332 (2009)

[16] S. Rosswog, E. Ramirez-Ruiz, R. Hix, Astrophys. J. 695, 404 (2009), 0808. 2143

[17] M.A. Abramowicz, B. Czerny, J.P. Lasota, E. Szuszkiewicz, Ap. J. 332, 646 (1988)
[18] J.P. Lasota, Slim accretion disks, in Theory of Accretion Disks, edited by W.J.D. et al. (Garching, 1994), Vol. 417 of NATO ASI Series C, p. 341

[19] T. Kawaguchi, Ap. J. 593, 69 (2003)

[20] A. Sadowski, Ap. J. Supp. 183, 171 (2009) 